\title{
Enzyme-agnostic lysosomal screen identifies new legumain-cleavable ADC linkers
}

Jared T. Miller, Caitlin N. Vitro, Siteng Fang, Samantha R. Benjamin, and L. Nathan Tumey*

AUTHOR ADDRESS School of Pharmacy and Pharmaceutical Sciences, Binghamton University, P.O. Box 6000, Binghamton NY 13902. Phone: 607-777-5844. Email: NTumey@binghamton.edu.

\section{Supporting Information}

List of supporting information in this document:

- Figure S1. Michael addition of TAMRA-thiol to the N-terminal maleimide fluorescence kinetics showing the rapid FRET quenching.

- Figure S2. Cleavage of ValCit and D-Val-D-Cit by cathepsin B.

- Figure S3. Oregon Green fluorescent Standard curve (double log) showing linearity from 3.2nM to $10 \mu \mathrm{M} . \mathrm{R}=0.9989$.

- Figure S4. Cytotoxicity curves against Ramos cells.

- Figure S5. Cytotoxicity curves against SKBR3 cells.

- Figure S6. Cleavage of select Asn-containing FRET pairs by legumain at various $\mathrm{pH}$.

- Figure S7. Synthesis of Asparagine-Based Linker ADCs

- Table S1a. Analytical Characterization and Reaction Results of Mal-AEEA-Gly-P2-P1-paminobenzyl amide Oregon Green Synthesis. (compounds 4)

- Table S1b. Analytical Characterization and Reaction Results of Mal-AEEA-Gly-His-P1-paminobenzyl amide Oregon Green Synthesis.

- Table S1c. Analytical Characterization and Reaction Results of Mal-AEEA-Gly-P2-His- paminobenzyl amide Oregon Green Synthesis.

- Table S1d. Analytical Characterization and Reaction Results of Mal-AEEA-Gly-P2-Lys- paminobenzyl amide Oregon Green Synthesis.

- Table S2. Percent Oregon Green Fluorescence Signal Quenching of FRET Pairs upon addition of the TAMRA-thiol.

- Table S3. Table of full data set of FRET library screening results. [Microsoft Excel attachment]

- Table S4. Analytical Characterization and Reaction Results of Mal-AEEA-Gly-P2-P1-PAB-OH Synthesis.

- Table S5. Analytical Characterization and Results of Mal-AEEA-Gly-P2-P1-PABC-MMAE Synthesis.

- Table S6. Characterization of ADCs.

- Table S7: Cytotoxicity data for ADCs showing the calculated error range

- Table S8. Initial Enzymatic Velocities and Regression Analysis of Catabolic Enzymes against Linkers

- LCMS methods (analytical) 


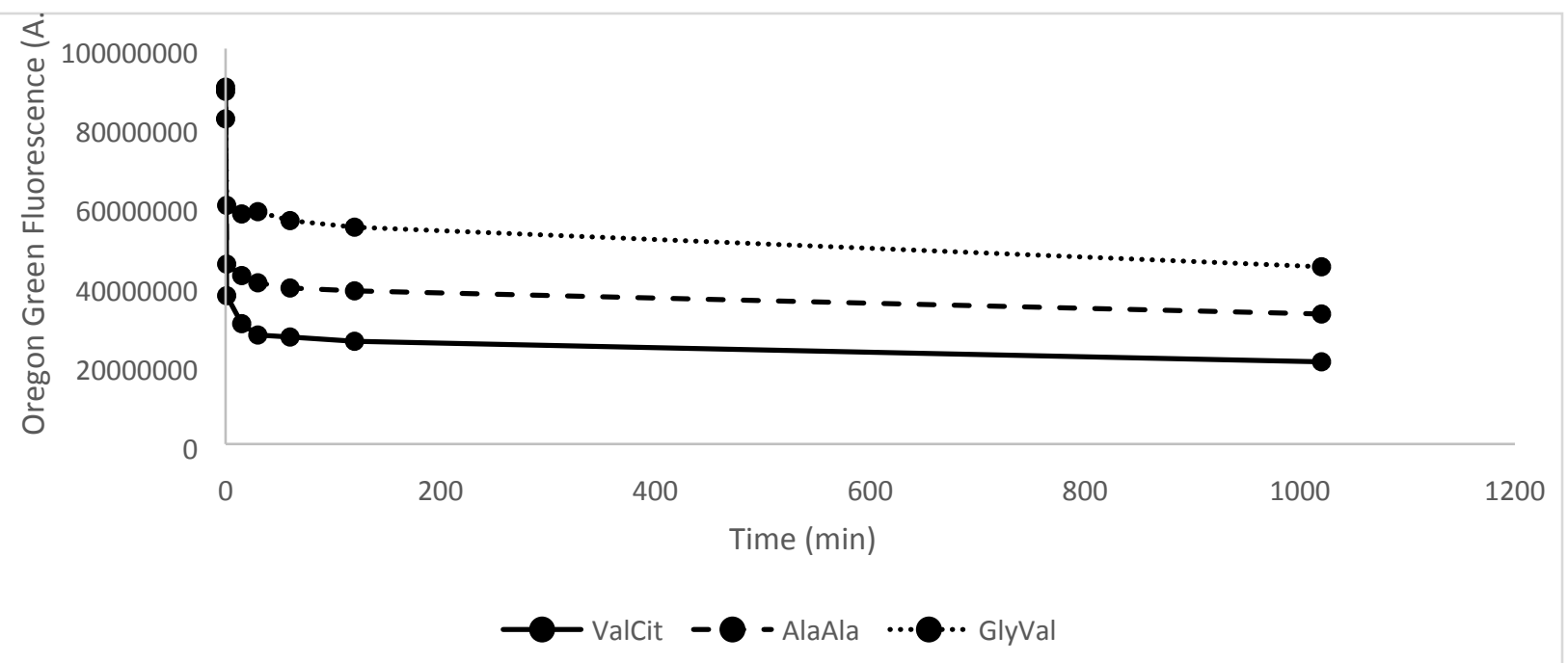

Figure S1. Michael addition of TAMRA-thiol to the N-terminal maleimide fluorescence kinetics showing the rapid FRET quenching.

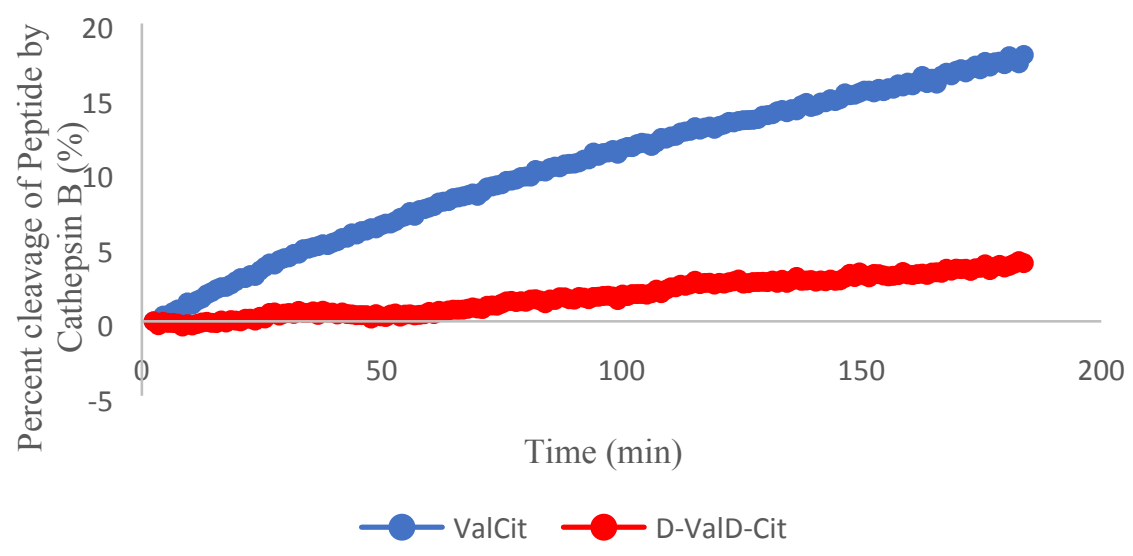

Figure S2. Cleavage of ValCit and D-Val-D-Cit by cathepsin B. 


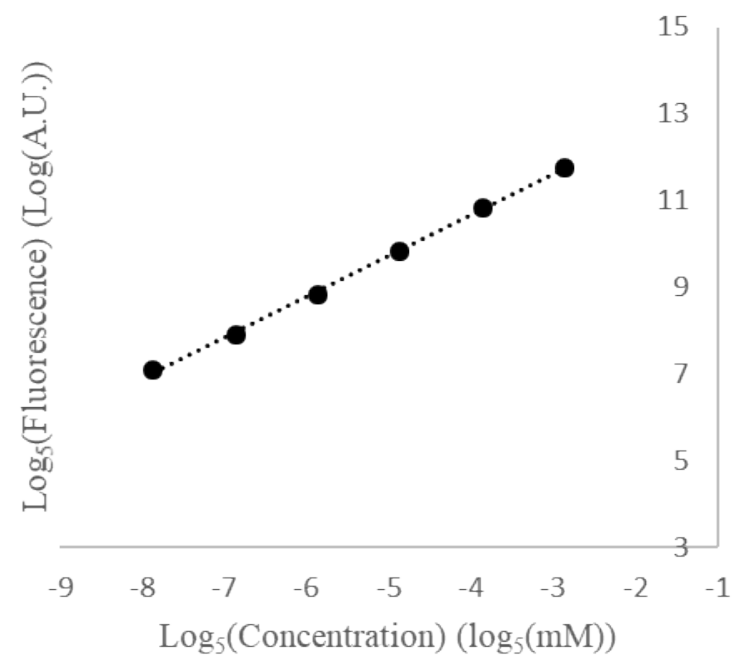

Figure S3. Oregon Green fluorescent Standard curve (double log) showing linearity from $3.2 \mathrm{nM}$ to $10 \mu \mathrm{M} . \mathrm{R}=0.9989$. 

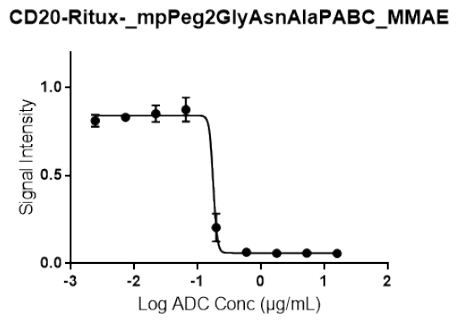

CD20-Ritux-_mpPeg2GlyGInVaIPABC_MMAE

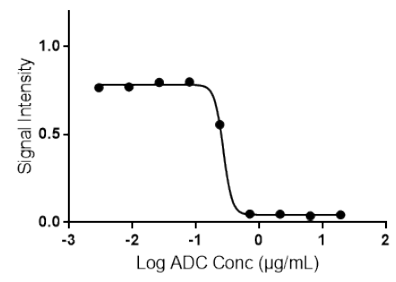

CD20-Ritux-_mpPeg2GlyValCitPABC_MMAE

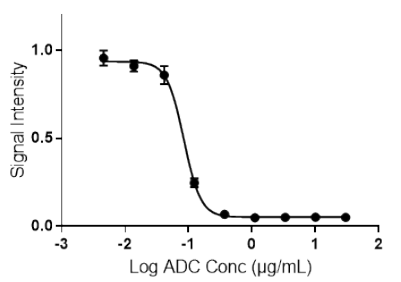

Her2-Tras-_mpPeg2GlyGInAsnPABC_MMAE

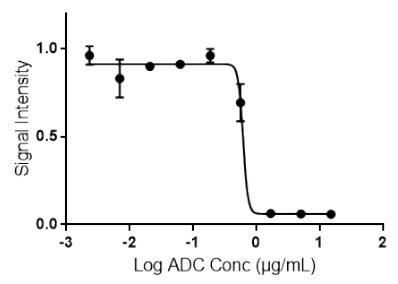

Her2-Tras-_mpPeg2GlyAsnAibPABC_MMAE

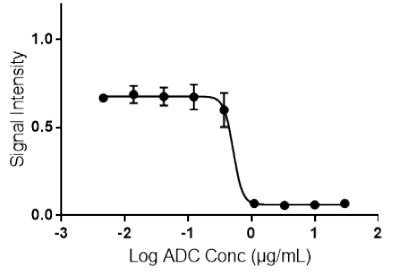

CD20-Ritux-_mpPeg2GlyAsnAsnPABC_MMAE

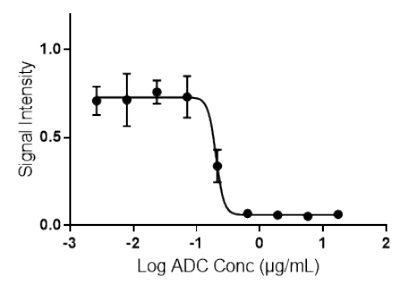

CD20-Ritux-_mpPeg2GlyTyrTrpPABC_MMAE

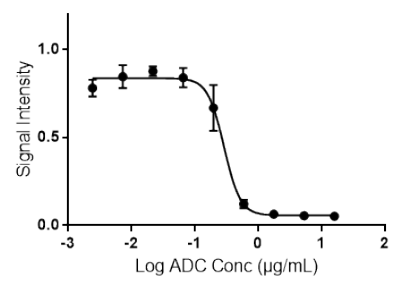

Her2-Tras-_mpPeg2GlyAsnAlaPABC_MMAE

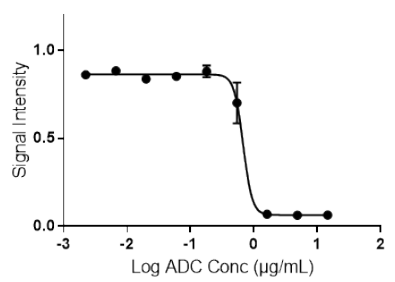

Her2-Tras-_mpPeg2GlyGInValPABC_MMAE

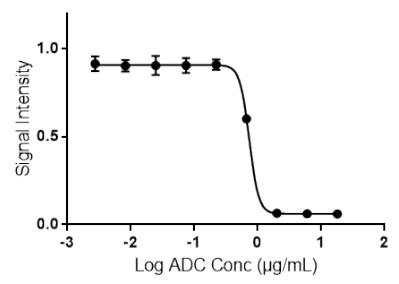

Her2-Tras-_mpPeg2GlyValCitPABC_MMAE

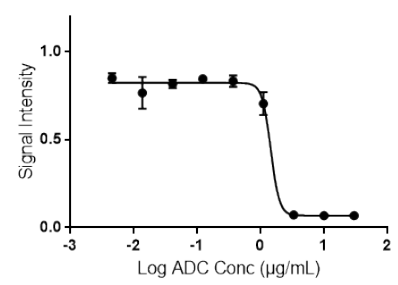

CD20-Ritux-_mpPeg2GlyGInAsnPABC_MMAE

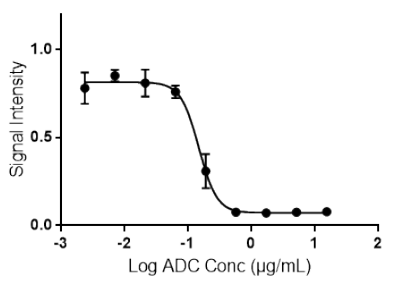

CD20-Ritux-_mpPeg2GlyAsnAibPABC_MMAE

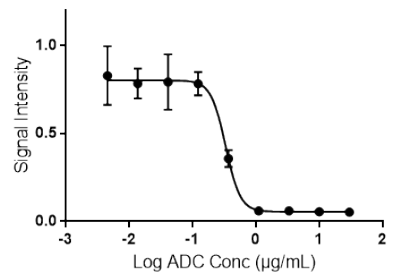

Her2-Tras-_mpPeg2GlyAsnAsnPABC_MMAE

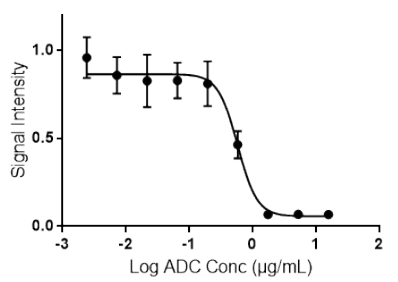

Her2-Tras-_mpPeg2Gly TyrTrpPABC_MMAE

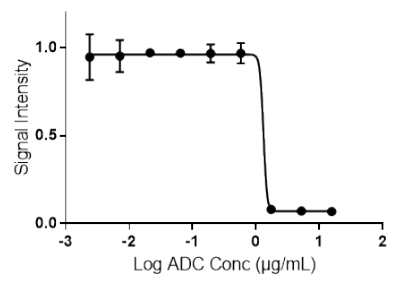

Figure S4. Cytotoxicity curves against Ramos cells. 
CD20-Ritux-_mpPeg2GlyAsnAlaPABC_MMAE

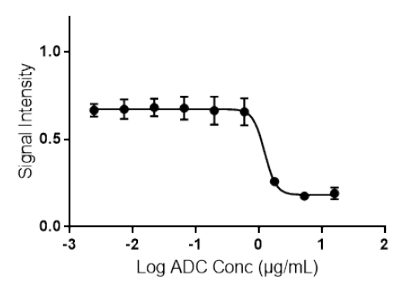

CD20-Ritux-_mpPeg2GlyGInValPABC_MMAE

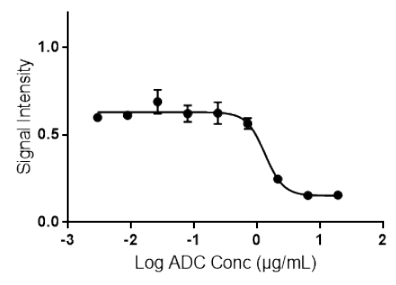

CD20-Ritux-_mpPeg2GlyValCitPABC_MMAE

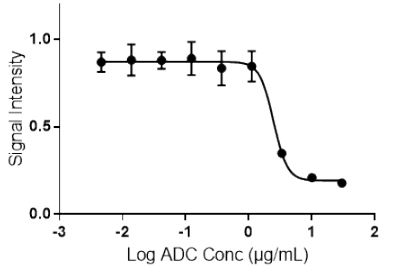

Her2-Tras-_mpPeg2GlyGInAsnPABC_MMAE

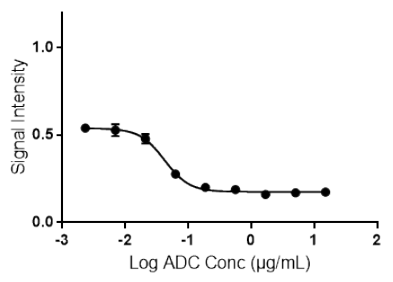

Her2-Tras-_mpPeg2GlyAsnAibPABC_MMAE

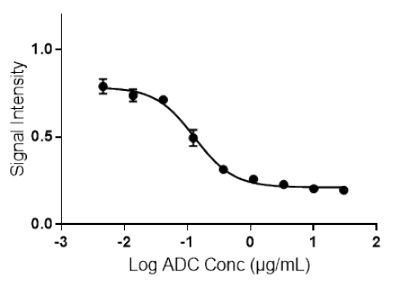

CD20-Ritux-_mpPeg2GlyAsnAsnPABC_MMAE

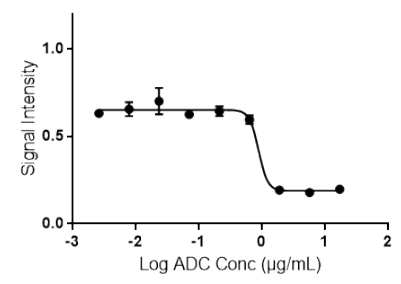

CD20-Ritux-_mpPeg2GlyGInAsnPABC_MMAE

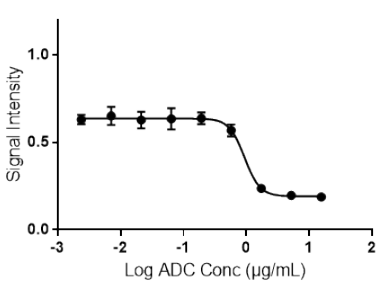

CD20-Ritux-_mpPeg2Gly TyrTrpPABC_MMAE

CD20-Ritux-_mpPeg2GlyAsnAibPABC_MMAE
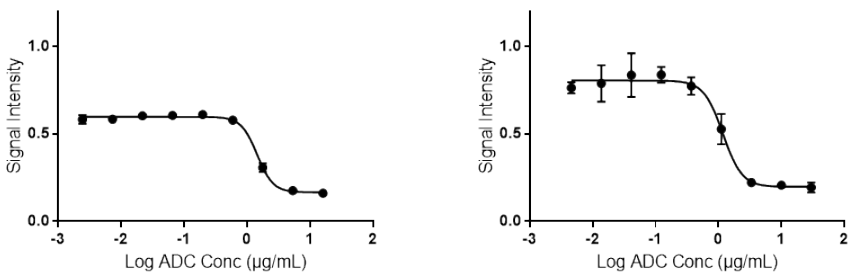

Her2-Tras-_mpPeg2GlyAsnAlaPABC_MMAE

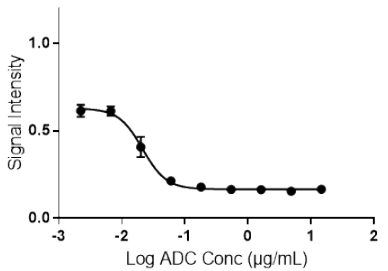

Her2-Tras-_mpPeg2GlyAsnAsnPABC_MMAE

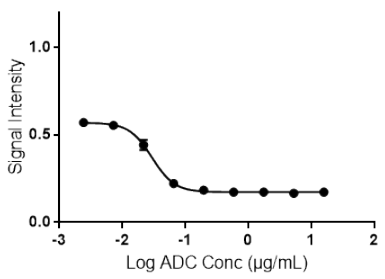

Her2-Tras-_mpPeg2GlyGInValPABC_MMAE

Her2-Tras-_mpPeg2GlyTyrTrpPABC_MMAE
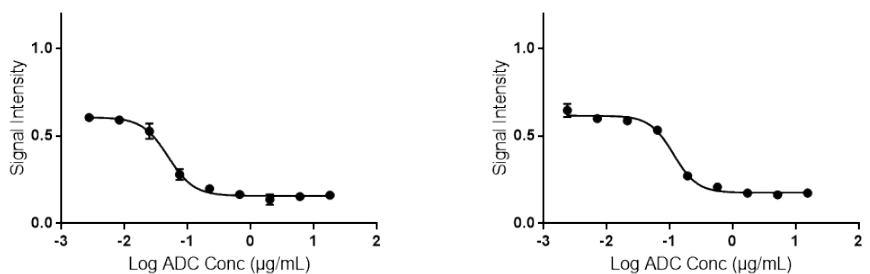

Her2-Tras-_mpPeg2GlyValCitPABC_MMAE

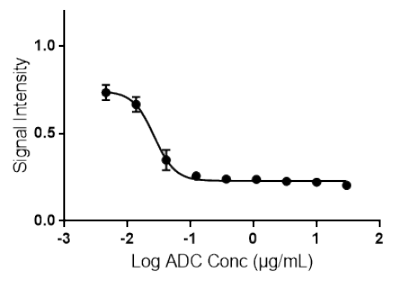

Figure S5. Cytotoxicity curves against SKBR3 cells. 
A)

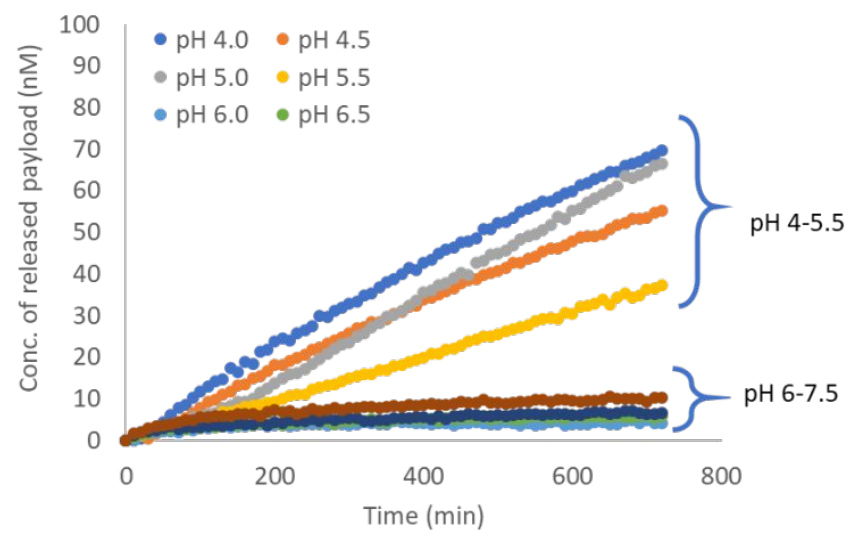

B)

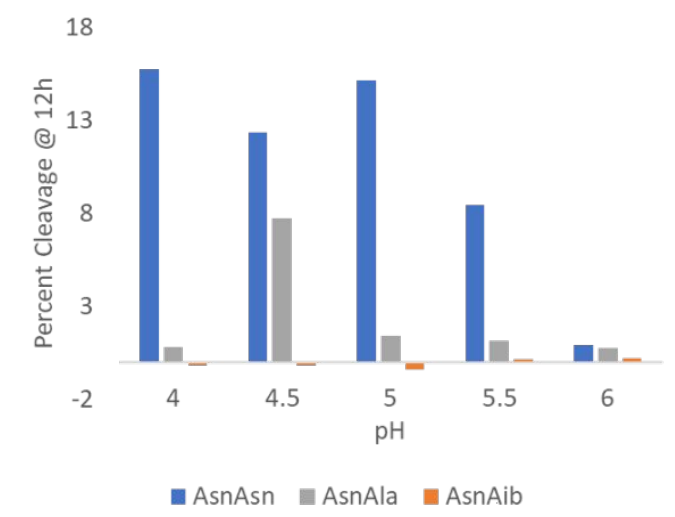

Figure S6. Cleavage of select Asn-containing FRET pairs by legumain at various $\mathrm{pH}$.

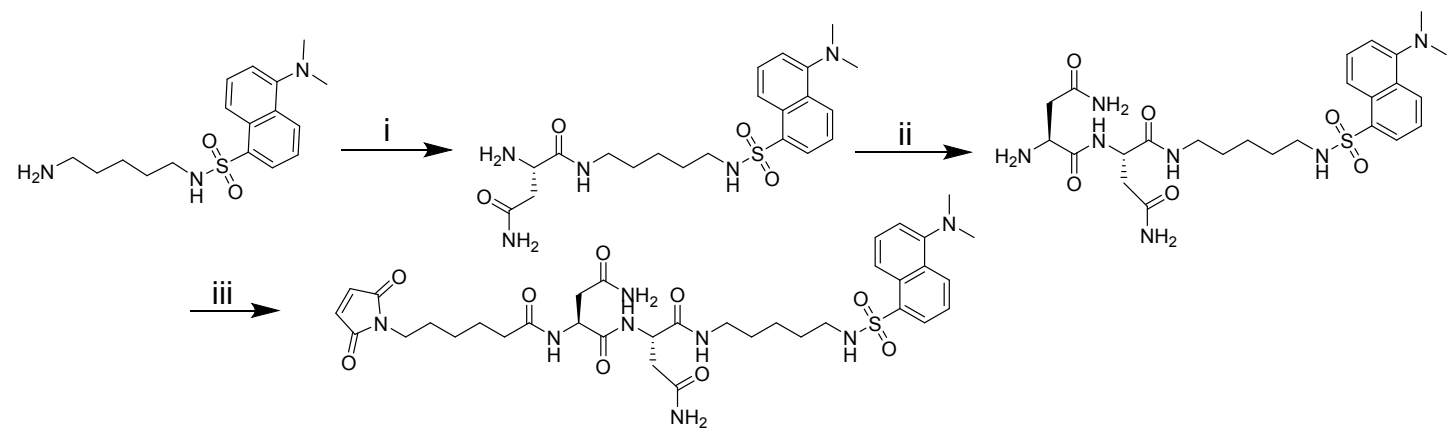

i. a) Boc-L-Asparagine (1.5eq), HATU (4eq), HOBt (1eq), 2,6-Lutidine (4eq), DMA, b) 20\% TFA in DCM ii. a) Boc-L-Asparagine (1.5eq), HATU (4eq), HOBt (1eq), 2,6-Lutidine (4eq), DMA, b) 20\% TFA in DCM iii. NSuccinimidyl 3-maleimidohexanoate (1.5eq), DIPEA (5eq.)

Figure S7. Synthesis of mcAsnAsn_Dansyl Cadaverine

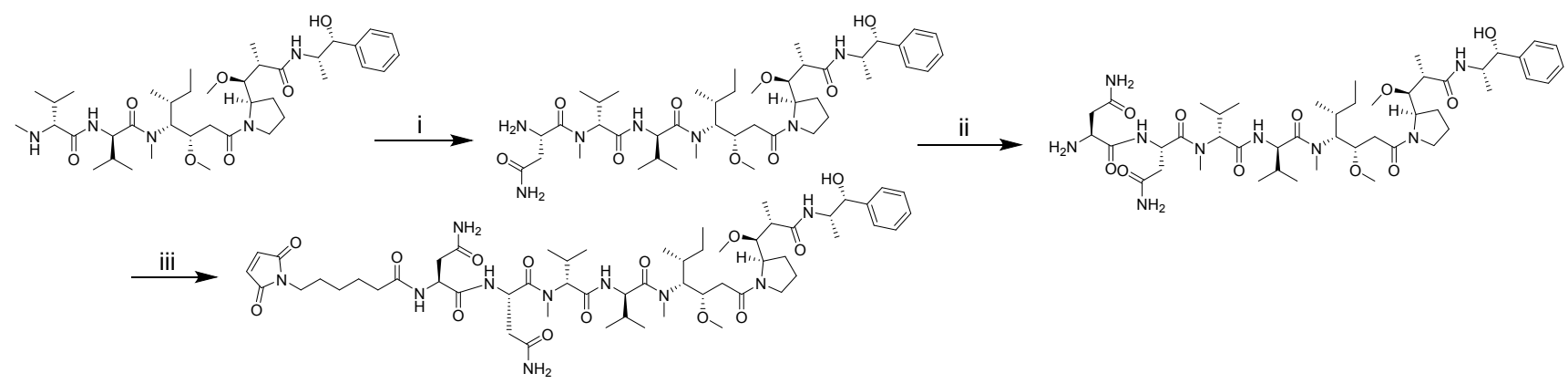

i. a) Boc-L-Asn(Trt)-OH (1.5eq), HATU (4eq), HOBt (1eq), 2,6-Lutidine (4eq), DMF, b)

TFA:Triethylsilane:water (95:2.5:2.5) ii. a) Boc-L-Asn(Trt)-OH (1.5eq), HATU (4eq), HOBt (1eq), 2,6Lutidine (4eq), DMF, b) TFA:Triethylsilane:water (95:2.5:2.5) iii. N-Succinimidyl 3-maleimidohexanoate (1.5eq), DIPEA (5eq.)

Figure S8. Synthesis of mcAsnAsn_MMAE 
Table S1a. Analytical Characterization and Reaction Results of Mal-AEEA-Gly- $\mathrm{P}_{2}-\mathrm{P}_{1}$-p-aminobenzyl amide Oregon Green Synthesis. (compounds 4)

\begin{tabular}{|c|c|c|c|c|c|}
\hline $\begin{array}{l}\text { Product Linker Chemistry } \\
\text { (Mal-AEEA-Gly-AA1-AA2-PABA-OG) }\end{array}$ & $\begin{array}{l}\text { ESI: } \\
(\mathrm{M}+\mathrm{H})^{+}\end{array}$ & $\begin{array}{l}\text { Retention } \\
\text { Time (min) }\end{array}$ & $\begin{array}{l}\text { Mass Recovered } \\
\text { (mg) }\end{array}$ & $\begin{array}{l}\text { Percent } \\
\text { Yield (\%) }\end{array}$ & $\begin{array}{l}\text { HPLC } \\
\text { Purity }\end{array}$ \\
\hline 3-Mal-AEEA-Gly-Val-Cit-PABA-OG & 1126.53 & 2.91 & 0.072 & 6.41 & $>99 \%$ \\
\hline $\begin{array}{l}\text { 3-Mal-AEEA-Gly-D-Val-D-Cit-PABA- } \\
\text { OG }\end{array}$ & 1126.56 & 2.90 & 0.156 & 13.79 & $>99 \%$ \\
\hline 3-Mal-AEEA-Gly-Ala-Ala-PABA-OG & 1012.43 & 2.88 & 0.186 & 18.38 & $>99 \%$ \\
\hline 3-Mal-AEEA-Gly-Gly-Gly-PABA-OG & 984-.39 & 2.80 & 0.247 & 20.60 & $>95 \%$ \\
\hline 3-Mal-AEEA-Gly-Gly-Asn-PABA-OG & 1041.47 & 2.75 & 0.062 & 4.85 & $>99 \%$ \\
\hline 3-Mal-AEEA-Gly-Gly-Aib-PABA-OG & 1012.40 & 2.88 & 0.044 & 3.56 & $\sim 60 \%$ \\
\hline 3-Mal-AEEA-Gly-Gly-1nal-PABA-OG & 1124.48 & 3.28 & 0.213 & 15.52 & $>99 \%$ \\
\hline 3-Mal-AEEA-Gly-Gly-2nal-PABA-OG & 1124.51 & 3.30 & 0.163 & 11.92 & $>99 \%$ \\
\hline 3-Mal-AEEA-Gly-Gly-Phe-PABA-OG & 1074.52 & 3.11 & 0.131 & 10.03 & $\sim 65 \%$ \\
\hline 3-Mal-AEEA-Gly-Gly-Val-PABA-OG & 1026.49 & 3.00 & 0.121 & 9.71 & $\sim 65 \%$ \\
\hline 3-Mal-AEEA-Gly-Gly-Cit-PABA-OG & 1084.47 & 2.76 & 0.133 & 10.05 & $\sim 75 \%$ \\
\hline 3-Mal-AEEA-Gly-Gly-Trp-PABA-OG & 1113.51 & 3.11 & 0.084 & 6.19 & $\sim 80 \%$ \\
\hline 3-Mal-AEEA-Gly-Ser-Asn-PABA-OG & 1071.45 & 2.75 & 0.126 & 9.65 & $>99 \%$ \\
\hline 3-Mal-AEEA-Gly-Ser-Aib-PABA-OG & 1042.46 & 2.86 & 0.167 & 13.20 & $>99 \%$ \\
\hline 3-Mal-AEEA-Gly-Ser-Phe-PABA-OG & 1104.55 & 3.10 & 0.547 & 40.73 & $>95 \%$ \\
\hline 3-Mal-AEEA-Gly-Ser-Ala-PABA-OG & 1028.44 & 2.83 & 0.273 & 21.78 & $>99 \%$ \\
\hline 3-Mal-AEEA-Gly-Ser-Val-PABA-OG & 1056.53 & 2.96 & 0.366 & 28.48 & $>99 \%$ \\
\hline 3-Mal-AEEA-Gly-Ser-Cit-PABA-OG & 1114.55 & 2.75 & 0.266 & 19.58 & $>99 \%$ \\
\hline 3-Mal-AEEA-Gly-Ser-Trp-PABA-OG & 1143.52 & 3.09 & 0.312 & 22.42 & $>99 \%$ \\
\hline 3-Mal-AEEA-Gly-Pro-Asn-PABA-OG & 1081.50 & 2.81 & 0.171 & 12.97 & $>92 \%$ \\
\hline 3-Mal-AEEA-Gly-Pro-Aib-PABA-OG & 1052.51 & 2.86 & 0.149 & 11.66 & $>60 \%$ \\
\hline 3-Mal-AEEA-Gly-Pro-Phe-PABA-OG & 1114.54 & 3.21 & 0.367 & 27.05 & $>95 \%$ \\
\hline 3-Mal-AEEA-Gly-Pro-Ala-PABA-OG & 1038.46 & 2.91 & 0.389 & 30.79 & $>92 \%$ \\
\hline 3-Mal-AEEA-Gly-Pro-Val-PABA-OG & 1066.50 & 3.06 & 0.361 & 27.80 & $>99 \%$ \\
\hline 3-Mal-AEEA-Gly-Pro-Cit-PABA-OG & 1124.53 & 2.83 & 0.290 & 21.21 & $>92 \%$ \\
\hline 3-Mal-AEEA-Gly-Pro-Trp-PABA-OG & 1153.50 & 3.18 & 0.245 & 17.43 & $>99 \%$ \\
\hline 3-Mal-AEEA-Gly-Tyr-Asn-PABA-OG & 1147.48 & 2.85 & 0.164 & 11.75 & $>99 \%$ \\
\hline 3-Mal-AEEA-Gly-Tyr-Aib-PABA-OG & 1118.51 & 3.00 & 0.072 & 5.29 & $>99 \%$ \\
\hline 3-Mal-AEEA-Gly-Tyr-Phe-PABA-OG & 1180.50 & 3.20 & 0.282 & 19.63 & $>90 \%$ \\
\hline 3-Mal-AEEA-Gly-Tyr-Ala-PABA-OG & 1104.48 & 2.95 & 0.320 & 23.82 & $>99 \%$ \\
\hline 3-Mal-AEEA-Gly-Tyr-Val-PABA-OG & 1132.53 & 3.06 & 0.241 & 17.46 & $>90 \%$ \\
\hline 3-Mal-AEEA-Gly-Tyr-Cit-PABA-OG & 1190.91 & 2.86 & 0.256 & 17.67 & $>90 \%$ \\
\hline 3-Mal-AEEA-Gly-Tyr-Trp-PABA-OG & 1219.45 & 3.18 & 0.195 & 13.15 & $>99 \%$ \\
\hline 3-Mal-AEEA-Gly-GIn-Asn-PABA-OG & 1112.54 & 2.73 & 0.130 & 9.57 & $>99 \%$ \\
\hline 3-Mal-AEEA-Gly-GIn-Aib-PABA-OG & 1083.51 & 2.85 & 0.099 & 7.54 & $>95 \%$ \\
\hline 3-Mal-AEEA-Gly-Gln-Phe-PABA-OG & 1145.52 & 3.06 & 0.305 & 21.87 & $>99 \%$ \\
\hline 3-Mal-AEEA-Gly-Gln-Ala-PABA-OG & 1069.45 & 2.81 & 0.471 & 36.16 & $>92 \%$ \\
\hline 3-Mal-AEEA-Gly-Gln-Val-PABA-OG & 1097.52 & 2.93 & 0.449 & 33.63 & $>80 \%$ \\
\hline 3-Mal-AEEA-Gly-Gln-Cit-PABA-OG & 1155.48 & 2.73 & 0.287 & 20.40 & $>99 \%$ \\
\hline 3-Mal-AEEA-Gly-Gln-Trp-PABA-OG & 1184.46 & 3.06 & 0.072 & 5.02 & $>99 \%$ \\
\hline 3-Mal-AEEA-Gly-Ile-Asn-PABA-OG & 1097.51 & 2.95 & 0.116 & 8.67 & $>95 \%$ \\
\hline
\end{tabular}




\begin{tabular}{|l|l|l|l|l|l|}
\hline 3-Mal-AEEA-Gly-Ile-Aib-PABA-OG & 1068.57 & 3.13 & 0.114 & 11.13 & $>99 \%$ \\
\hline 3-Mal-AEEA-Gly-Ile-Phe-PABA-OG & 1130.57 & 3.33 & 0.240 & 17.44 & $>95 \%$ \\
\hline 3-Mal-AEEA-Gly-Ile-Ala-PABA-OG & 1054.48 & 3.06 & 0.293 & 22.82 & $>99 \%$ \\
\hline 3-Mal-AEEA-Gly-Ile-Val-PABA-OG & 1082.55 & 3.20 & 0.236 & 17.89 & $>99 \%$ \\
\hline 3-Mal-AEEA-Gly-Ile-Cit-PABA-OG & 1140.51 & 2.96 & 0.163 & 11.71 & $>99 \%$ \\
\hline 3-Mal-AEEA-Gly-Ile-Trp-PABA-OG & 1169.53 & 3.30 & 0.140 & 9.81 & $>99 \%$ \\
\hline 3-Mal-AEEA-Gly-Asn-Asn-PABA-OG & 1098.47 & 2.73 & 0.165 & 12.32 & $>99 \%$ \\
\hline 3-Mal-AEEA-Gly-Asn-Aib-PABA-OG & 1069.46 & 2.83 & 0.188 & 14.45 & $>95 \%$ \\
\hline 3-Mal-AEEA-Gly-Asn-Phe-PABA-OG & 1131.49 & 3.05 & 0.348 & 25.24 & $>99 \%$ \\
\hline 3-Mal-AEEA-Gly-Asn-Ala-PABA-OG & 1055.53 & 2.81 & 0.200 & 15.61 & $>85 \%$ \\
\hline 3-Mal-AEEA-Gly-Asn-Val-PABA-OG & 1083.51 & 2.93 & 0.218 & 16.52 & $>99 \%$ \\
\hline 3-Mal-AEEA-Gly-Asn-Cit-PABA-OG & 1141.46 & 2.73 & 0.536 & 38.60 & $>99 \%$ \\
\hline 3-Mal-AEEA-Gly-Asn-Trp-PABA-OG & 1170.50 & 3.05 & 0.218 & 15.32 & $>99 \%$ \\
\hline
\end{tabular}

Table S1b. Analytical Characterization and Reaction Results of Mal-AEEA-Gly-His-P ${ }_{1}$-p-aminobenzyl amide Oregon Green Synthesis.

\begin{tabular}{|l|l|l|l|l|l|}
\hline $\begin{array}{l}\text { Product Linker Chemistry } \\
\text { (Mal-AEEA-Gly-AA1-AA2-PABA-OG) }\end{array}$ & $\begin{array}{l}\text { ESI: } \\
(\mathrm{M}+\mathrm{H})^{+}\end{array}$ & $\begin{array}{l}\text { Retention } \\
\text { Time }(\mathrm{min})\end{array}$ & $\begin{array}{l}\text { Mass Recovered } \\
(\mathrm{mg})\end{array}$ & $\begin{array}{l}\text { Percent } \\
\text { Yield }(\%)\end{array}$ & $\begin{array}{l}\text { HPLC } \\
\text { Purity }\end{array}$ \\
\hline 3-Mal-AEEA-Gly-His-Asn-PABA-OG & 1121.53 & 2.48 & 0.152 & 14.83 & $>99 \%$ \\
\hline 3-Mal-AEEA-Gly-His-Aib-PABA-OG & 1092.53 & 2.56 & 0.037 & 3.68 & $>99 \%$ \\
\hline 3-Mal-AEEA-Gly-His-Phe-PABA-OG & 1154.51 & 2.75 & 0.048 & 4.52 & $>99 \%$ \\
\hline 3-Mal-AEEA-Gly-His-Ala-PABA-OG & 1078.51 & 2.55 & 0.058 & 5.90 & $>99 \%$ \\
\hline 3-Mal-AEEA-Gly-His-Val-PABA-OG & 1106.53 & 2.85 & 0.042 & 4.20 & $>99 \%$ \\
\hline 3-Mal-AEEA-Gly-His-Cit-PABA-OG & 1164.55 & 2.48 & 0.031 & 2.92 & $>99 \%$ \\
\hline 3-Mal-AEEA-Gly-His-Trp-PABA-OG & 1193.50 & 2.76 & 0.039 & 3.58 & $>99 \%$ \\
\hline
\end{tabular}

Table S1c. Analytical Characterization and Reaction Results of Mal-AEEA-Gly- $\mathrm{P}_{2}-\mathrm{His}-\mathrm{p}$-aminobenzyl amide Oregon Green Synthesis.

\begin{tabular}{|l|l|l|l|l|l|}
\hline $\begin{array}{l}\text { Product Linker Chemistry } \\
\text { (3-Mal-AEEA-Gly-AA1-AA2-PABA-OG) }\end{array}$ & $\begin{array}{l}\text { ESI: } \\
(\mathrm{M}+\mathrm{H})^{+}\end{array}$ & $\begin{array}{l}\text { Retention } \\
\text { Time }(\mathrm{min})\end{array}$ & $\begin{array}{l}\text { Mass } \\
\text { Recovered } \\
(\mathrm{mg})\end{array}$ & $\begin{array}{l}\text { Percent } \\
\text { Yield (\%) }\end{array}$ & $\begin{array}{l}\text { HPLC } \\
\text { Purity }\end{array}$ \\
\hline 3-Mal-AEEA-Gly-Gly-His-PABA-OG & 1064.50 & 2.50 & 0.137 & 10.54 & $>99 \%$ \\
\hline 3-Mal-AEEA-Gly-Ser-His-PABA-OG & 1094.53 & 2.50 & 0.082 & 8.47 & $>99 \%$ \\
\hline 3-Mal-AEEA-Gly-Pro-His-PABA-OG & 1104.47 & 2.55 & 0.182 & 18.18 & $>99 \%$ \\
\hline 3-Mal-AEEA-Gly-Tyr-His-PABA-OG & 1170.50 & 2.58 & 0.144 & 14.28 & $>99 \%$ \\
\hline 3-Mal-AEEA-Gly-Gln-His-PABA-OG & 1135.51 & 2.48 & 0.213 & 19.97 & $>99 \%$ \\
\hline 3-Mal-AEEA-Gly-Ile-His-PABA-OG & 1120.53 & 2.66 & 0.102 & 9.86 & $>99 \%$ \\
\hline 3-Mal-AEEA-Gly-Asn-His-PABA-OG & 1121.51 & 2.48 & 0.119 & 11.65 & $>99 \%$ \\
\hline
\end{tabular}


Table S1d. Analytical Characterization and Reaction Results of Mal-AEEA-Gly-P2-Lys- $p$-aminobenzyl amide Oregon Green Synthesis.

\begin{tabular}{|l|l|l|l|l|l|}
\hline $\begin{array}{l}\text { Product Linker Chemistry (3- } \\
\text { Mal-AEEA-Gly-AA1-AA2-PABA-OG) }\end{array}$ & $\begin{array}{l}\text { ESI: } \\
(\mathrm{M}+\mathrm{H})^{+}\end{array}$ & $\begin{array}{l}\text { Retention } \\
\text { Time }(\mathrm{min})\end{array}$ & $\begin{array}{l}\text { Mass Recovered } \\
(\mathrm{mg})\end{array}$ & $\begin{array}{l}\text { Percent } \\
\text { Yield }(\%)\end{array}$ & $\begin{array}{l}\text { HPLC } \\
\text { Purity }\end{array}$ \\
\hline 3-Mal-AEEA-Gly-Gly-Lys-PABA-OG & 1055.55 & 2.50 & 0.120 & 9.30 & $>99 \%$ \\
\hline 3-Mal-AEEA-Gly-Ser-Lys-PABA-OG & 1085.61 & 2.50 & 0.009 & 0.70 & $>70 \%$ \\
\hline 3-Mal-AEEA-Gly-Pro-Lys-PABA-OG & 1095.59 & 2.55 & 0.102 & 7.64 & $>99 \%$ \\
\hline 3-Mal-AEEA-Gly-Tyr-Lys-PABA-OG & 1161.11 & 2.51 & 0.338 & 23.93 & $>50 \%$ \\
\hline 3-Mal-AEEA-Gly-Gln-Lys-PABA-OG & 1126.52 & 2.46 & 0.137 & 9.99 & $>99 \%$ \\
\hline 3-Mal-AEEA-Gly-Ile-Lys-PABA-OG & 1111.58 & 2.70 & 0.208 & 15.36 & $>60 \%$ \\
\hline 3-Mal-AEEA-Gly-Asn-Lys-PABA-OG & 1112.56 & 2.46 & 0.164 & 12.14 & $>99 \%$ \\
\hline
\end{tabular}

Table S2. Percent Oregon Green Fluorescence Signal Quenching of FRET Pairs upon addition of the TAMRA-thiol.

\begin{tabular}{|c|c|}
\hline FRET Pair Chemistry (AA1-AA2) & $\begin{array}{c}\text { Percent OG Signal } \\
\text { Quenched (\%) }\end{array}$ \\
\hline TAMRA-3-Mal-AEEA-Gly-Val-Cit-PABA-OG & 83.3 \\
\hline TAMRA-3-Mal-AEEA-Gly-D-Val-D-Cit-PABA-OG & 74.6 \\
\hline TAMRA-3-Mal-AEEA-Gly-Ala-Ala-PABA-OG & 54.0 \\
\hline TAMRA-3-Mal-AEEA-Gly-Gly-Gly-PABA-OG & 77.0 \\
\hline TAMRA-3-Mal-AEEA-Gly-Gly-Asn-PABA-OG & 61.5 \\
\hline TAMRA-3-Mal-AEEA-Gly-Gly-Aib-PABA-OG & 48.5 \\
\hline TAMRA-3-Mal-AEEA-Gly-Gly-1nal-PABA-OG & 78.8 \\
\hline TAMRA-3-Mal-AEEA-Gly-Gly-2nal-PABA-OG & 76.4 \\
\hline TAMRA-3-Mal-AEEA-Gly-Gly-Phe-PABA-OG & 36.9 \\
\hline TAMRA-3-Mal-AEEA-Gly-Gly-His-PABA-OG & 74.7 \\
\hline TAMRA-3-Mal-AEEA-Gly-Gly-Val-PABA-OG & 37.7 \\
\hline TAMRA-3-Mal-AEEA-Gly-Gly-Lys-PABA-OG & 36.6 \\
\hline TAMRA-3-Mal-AEEA-Gly-Gly-Cit-PABA-OG & 40.6 \\
\hline TAMRA-3-Mal-AEEA-Gly-Gly-Trp-PABA-OG & 42.5 \\
\hline TAMRA-3-Mal-AEEA-Gly-Ser-Asn-PABA-OG & 86.3 \\
\hline TAMRA-3-Mal-AEEA-Gly-Ser-Aib-PABA-OG & 84.9 \\
\hline TAMRA-3-Mal-AEEA-Gly-Ser-Phe-PABA-OG & 67.7 \\
\hline TAMRA-3-Mal-AEEA-Gly-Ser-His-PABA-OG & 54.9 \\
\hline TAMRA-3-Mal-AEEA-Gly-Ser-Ala-PABA-OG & 77.5 \\
\hline TAMRA-3-Mal-AEEA-Gly-Ser-Val-PABA-OG & 70.8 \\
\hline TAMRA-3-Mal-AEEA-Gly-Ser-Lys-PABA-OG & 28.1 \\
\hline TAMRA-3-Mal-AEEA-Gly-Ser-Cit-PABA-OG & 84.5 \\
\hline TAMRA-3-Mal-AEEA-Gly-Ser-Trp-PABA-OG & 83.6 \\
\hline TAMRA-3-Mal-AEEA-Gly-Pro-Asn-PABA-OG & 75.2 \\
\hline TAMRA-3-Mal-AEEA-Gly-Pro-Aib-PABA-OG & 57.1 \\
\hline TAMRA-3-Mal-AEEA-Gly-Pro-Phe-PABA-OG & 75.0 \\
\hline TAMRA-3-Mal-AEEA-Gly-Pro-His-PABA-OG & 84.8 \\
\hline TAMRA-3-Mal-AEEA-Gly-Pro-Ala-PABA-OG & 76.9 \\
\hline
\end{tabular}




\begin{tabular}{|c|c|}
\hline TAMRA-3-Mal-AEEA-Gly-Pro-Val-PABA-OG & 86.9 \\
\hline TAMRA-3-Mal-AEEA-Gly-Pro-Lys-PABA-OG & 71.1 \\
\hline TAMRA-3-Mal-AEEA-Gly-Pro-Cit-PABA-OG & 77.2 \\
\hline TAMRA-3-Mal-AEEA-Gly-Pro-Trp-PABA-OG & 83.9 \\
\hline TAMRA-3-Mal-AEEA-Gly-His-Asn-PABA-OG & 83.5 \\
\hline TAMRA-3-Mal-AEEA-Gly-His-Aib-PABA-OG & 77.0 \\
\hline TAMRA-3-Mal-AEEA-Gly-His-Phe-PABA-OG & 71.4 \\
\hline TAMRA-3-Mal-AEEA-Gly-His-Ala-PABA-OG & 70.3 \\
\hline TAMRA-3-Mal-AEEA-Gly-His-Val-PABA-OG & 75.2 \\
\hline TAMRA-3-Mal-AEEA-Gly-His-Cit-PABA-OG & 69.8 \\
\hline TAMRA-3-Mal-AEEA-Gly-His-Trp-PABA-OG & 74.7 \\
\hline TAMRA-3-Mal-AEEA-Gly-Tyr-Asn-PABA-OG & 86.8 \\
\hline TAMRA-3-Mal-AEEA-Gly-Tyr-Aib-PABA-OG & 85.2 \\
\hline TAMRA-3-Mal-AEEA-Gly-Tyr-Phe-PABA-OG & 74.2 \\
\hline TAMRA-3-Mal-AEEA-Gly-Tyr-Phe-PABA-OG & 82.6 \\
\hline TAMRA-3-Mal-AEEA-Gly-Tyr-Ala-PABA-OG & 78.5 \\
\hline TAMRA-3-Mal-AEEA-Gly-Tyr-Val-PABA-OG & 72.7 \\
\hline TAMRA-3-Mal-AEEA-Gly-Tyr-Lys-PABA-OG & 25.0 \\
\hline TAMRA-3-Mal-AEEA-Gly-Tyr-Cit-PABA-OG & 70.0 \\
\hline TAMRA-3-Mal-AEEA-Gly-Tyr-Trp-PABA-OG & 84.7 \\
\hline TAMRA-3-Mal-AEEA-Gly-GIn-Asn-PABA-OG & 89.2 \\
\hline TAMRA-3-Mal-AEEA-Gly-Gln-Aib-PABA-OG & 79.8 \\
\hline TAMRA-3-Mal-AEEA-Gly-Gln-Phe-PABA-OG & 81.5 \\
\hline TAMRA-3-Mal-AEEA-Gly-Gln-His-PABA-OG & 87.0 \\
\hline TAMRA-3-Mal-AEEA-Gly-Gln-Ala-PABA-OG & 57.1 \\
\hline TAMRA-3-Mal-AEEA-Gly-Gln-Val-PABA-OG & 57.5 \\
\hline TAMRA-3-Mal-AEEA-Gly-Gln-Lys-PABA-OG & 43.8 \\
\hline TAMRA-3-Mal-AEEA-Gly-GIn-Cit-PABA-OG & 83.8 \\
\hline TAMRA-3-Mal-AEEA-Gly-Gln-Trp-PABA-OG & 88.1 \\
\hline TAMRA-3-Mal-AEEA-Gly-Ile-Asn-PABA-OG & 79.9 \\
\hline TAMRA-3-Mal-AEEA-Gly-Ile-Aib-PABA-OG & 92.3 \\
\hline TAMRA-3-Mal-AEEA-Gly-Ile-Phe-PABA-OG & 78.3 \\
\hline TAMRA-3-Mal-AEEA-Gly-Ile-His-PABA-OG & 83.6 \\
\hline TAMRA-3-Mal-AEEA-Gly-Ile-Ala-PABA-OG & 91.8 \\
\hline TAMRA-3-Mal-AEEA-Gly-Ile-Val-PABA-OG & 93.9 \\
\hline TAMRA-3-Mal-AEEA-Gly-lle-Lys-PABA-OG & 38.6 \\
\hline TAMRA-3-Mal-AEEA-Gly-Ile-Cit-PABA-OG & 88.6 \\
\hline TAMRA-3-Mal-AEEA-Gly-Ile-Trp-PABA-OG & 87.2 \\
\hline TAMRA-3-Mal-AEEA-Gly-Asn-Asn-PABA-OG & 88.7 \\
\hline TAMRA-3-Mal-AEEA-Gly-Asn-Aib-PABA-OG & 87.3 \\
\hline TAMRA-3-Mal-AEEA-Gly-Asn-Phe-PABA-OG & 87.1 \\
\hline TAMRA-3-Mal-AEEA-Gly-Asn-His-PABA-OG & 85.0 \\
\hline TAMRA-3-Mal-AEEA-Gly-Asn-Ala-PABA-OG & 71.5 \\
\hline TAMRA-3-Mal-AEEA-Gly-Asn-Val-PABA-OG & 81.6 \\
\hline TAMRA-3-Mal-AEEA-Gly-Asn-Lys-PABA-OG & 80.5 \\
\hline TAMRA-3-Mal-AEEA-Gly-Asn-Cit-PABA-OG & 90.9 \\
\hline
\end{tabular}


Table S3. Table of full data set of FRET library screening results. [Microsoft Excel attachment]

Table S4. Analytical Characterization and Reaction Results of Mal-AEEA-Gly- $\mathrm{P}_{2}-\mathrm{P}_{1}-\mathrm{PAB}-\mathrm{OH}$ Synthesis.

\begin{tabular}{|l|l|l|l|l|l|}
\hline $\begin{array}{l}\text { Product Linker Chemistry (3- } \\
\text { Mal-AEEA-Gly-AA1-AA2-PAB-OH) }\end{array}$ & $\begin{array}{l}\text { ESI: } \\
(\mathrm{M}+\mathrm{H})^{+}\end{array}$ & $\begin{array}{l}\text { Retention } \\
\text { Time }(\mathrm{min})\end{array}$ & $\begin{array}{l}\text { Mass Recovered } \\
(\mathrm{mg})\end{array}$ & $\begin{array}{l}\text { Percent } \\
\text { Yield }(\%)\end{array}$ & $\begin{array}{l}\text { HPLC } \\
\text { Purity }\end{array}$ \\
\hline 3-Mal-AEEA-Gly-Val-Cit-PAB-OH & 733.3 & 2.36 & 9.5 & 86.4 & $>99 \%$ \\
\hline 3-Mal-AEEA-Gly-Tyr-Trp-PAB-OH & 826.3 & 2.79 & 9.7 & 78.4 & $>99 \%$ \\
\hline 3-Mal-AEEA-Gly-GIn-Asn-PAB-OH & 719.2 & 2.08 & 7.4 & 68.7 & $>99 \%$ \\
\hline 3-Mal-AEEA-Gly-GIn-Val-PAB-OH & 704.3 & 2.42 & 9.2 & 87.2 & $>99 \%$ \\
\hline 3-Mal-AEEA-Gly-Asn-Asn-PAB-OH & 705.3 & 2.05 & 8.5 & 80.4 & $>99 \%$ \\
\hline 3-Mal-AEEA-Gly-Asn-Ala-PAB-OH & 662.3 & 2.18 & 5.5 & 55.4 & $>99 \%$ \\
\hline 3-Mal-AEEA-Gly-Asn-Aib-PAB-OH & & 2.42 & 5.7 & 42.0 & $>50 \%$ \\
\hline
\end{tabular}

Table S5. Analytical Characterization and Results of Mal-AEEA-Gly- $P_{2}-P_{1}-P A B C-M M A E$ Synthesis.

\begin{tabular}{|l|l|l|l|l|l|}
\hline $\begin{array}{l}\text { Product Linker Chemistry (3- } \\
\text { Mal-AEEA-Gly-AA1-AA2-PABC-MMAE) }\end{array}$ & $\begin{array}{c}\mathrm{ESI} \\
(\mathrm{M}+\mathrm{H})^{+}\end{array}$ & $\begin{array}{c}\text { Retention } \\
\text { Time (min) }\end{array}$ & $\begin{array}{c}\text { Mass } \\
\text { Recovered } \\
(\mathrm{mg})\end{array}$ & $\begin{array}{c}\text { Percent } \\
\text { Yield (\%) } \\
\text { over 2 steps }\end{array}$ & $\begin{array}{c}\text { HPLC } \\
\text { Purity }\end{array}$ \\
\hline 3-Mal-AEEA-Gly-Val-Cit-PABC-MMAE & 1477.4 & 3.41 & 1.1 & 5.7 & $>99 \%$ \\
\hline 3-Mal-AEEA-Gly-Tyr-Trp-PABC-MMAE & 1570.5 & 3.57 & 1.7 & 9.2 & $>93 \%$ \\
\hline 3-Mal-AEEA-Gly-GIn-Asn-PABC-MMAE & 1463.5 & 3.31 & 2.9 & 19.2 & $>99 \%$ \\
\hline 3-Mal-AEEA-Gly-GIn-Val-PABC-MMAE & 1448.4 & 3.45 & 1.7 & 9.0 & $>99 \%$ \\
\hline 3-Mal-AEEA-Gly-Asn-Asn-PABC-MMAE & 1449.3 & 3.32 & 4.0 & 22.9 & $>99 \%$ \\
\hline 3-Mal-AEEA-Gly-Asn-Ala-PABC-MMAE & 1406.4 & 3.38 & 2.3 & 19.7 & $>99 \%$ \\
\hline 3-Mal-AEEA-Gly-Asn-Aib-PABC-MMAE & 1420.7 & 3.44 & 1.0 & 8.3 & $>99 \%$ \\
\hline
\end{tabular}

Table S6. Characterization of ADCs.

\begin{tabular}{|l|l|l|l|l|l|}
\hline Antibody & Linker payload & DAR & $\begin{array}{l}\text { Mass shift } \\
\text { (observed) }\end{array}$ & $\begin{array}{l}\text { Mass shift } \\
\text { (theoretical) }\end{array}$ & \%Aggregation \\
\hline Anti-Her2 & mpPeg2GlyValCitPABC_MMAE & 3.8 & 1476.0 & 1476.3 & $<5 \%$ \\
\hline Anti-Her2 & mpPeg2GlyTyrTrpPABC_MMAE & 3 & 1574.5 & 1569.5 & $<5 \%$ \\
\hline Anti-Her2 & mpPeg2GlyGInAsnPABC_MMAE & 5.7 & 1466.2 & 1462.3 & $<5 \%$ \\
\hline Anti-Her2 & mpPeg2GlyGInVaIPABC_MMAE & 5.4 & 1452.3 & 1447.3 & $<5 \%$ \\
\hline Anti-Her2 & mpPeg2GlyAsnAsnPABC_MMAE & 6.4 & 1450.4 & 1448.4 & $<5 \%$ \\
\hline Anti-Her2 & mpPeg2GlyAsnAlaPABC_MMAE & 6.1 & 1404.4 & 1405.4 & $<5 \%$ \\
\hline Anti-Her2 & mpPeg2GlyAsnAibPABC_MMAE & 3.9 & 1413.0 & 1419.6 & $<5 \%$ \\
\hline Anti-CD20 & mpPeg2GlyVaICitPABC_MMAE & 3.4 & 1476.0 & 1476.3 & $<5 \%$ \\
\hline Anti-CD20 & mpPeg2GlyTyrTrpPABC_MMAE & 3.5 & 1570.5 & 1569.5 & $<5 \%$ \\
\hline Anti-CD20 & mpPeg2GlyGInAsnPABC_MMAE & 5.0 & 1460.3 & 1462.3 & $<5 \%$ \\
\hline Anti-CD20 & mpPeg2GlyGInVaIPABC_MMAE & 3.3 & 1446.3 & 1447.3 & $<5 \%$ \\
\hline Anti-CD20 & mpPeg2GlyAsnAsnPABC_MMAE & 8.0 & 1446.4 & 1448.4 & $<5 \%$ \\
\hline Anti-CD20 & mpPeg2GlyAsnAlaPABC_MMAE & 8.0 & 1404.4 & 1405.4 & $<5 \%$ \\
\hline Anti-CD20 & mpPeg2GlyAsnAibPABC_MMAE & 3.7 & 1422.0 & 1419.6 & $<5 \%$ \\
\hline
\end{tabular}


Table S7: Cytotoxicity data for ADCs showing the calculated error range

\begin{tabular}{|c|c|c|c|c|c|c|c|}
\hline \multirow[b]{2}{*}{$A D C$} & \multirow[b]{2}{*}{ Linker } & \multicolumn{3}{|c|}{ SKBR3 (Her2+++) } & \multicolumn{3}{|c|}{ Ramos (Her2-) } \\
\hline & & $\begin{array}{c}\mathrm{IC50} \\
(\mu \mathrm{g} / \mathrm{mL})\end{array}$ & $\begin{array}{l}\text { Upper } \\
\text { limit }\end{array}$ & $\begin{array}{l}\text { Lower } \\
\text { limit }\end{array}$ & $\begin{array}{c}\text { IC50 } \\
(\mu \mathrm{g} / \mathrm{mL})\end{array}$ & $\begin{array}{l}\text { Upper } \\
\text { limit }\end{array}$ & $\begin{array}{c}\text { Lower } \\
\text { limit }\end{array}$ \\
\hline Anti-Her2-a & AsnAsn & 0.030 & 0.031 & 0.029 & 0.58 & 0.646 & 0.527 \\
\hline Anti-Her2-b & AsnAla & 0.021 & 0.023 & 0.020 & 0.68 & 0.858 & 0.531 \\
\hline Anti-Her2-c & GlnAsn & 0.042 & 0.045 & 0.039 & 0.62 & NA & NA \\
\hline Anti-Her2-d & GlnVal & 0.049 & 0.053 & 0.046 & 0.74 & 0.833 & 0.656 \\
\hline Anti-Her2-e & TyrTrp & 0.115 & 0.123 & 0.108 & 1.31 & NA & NA \\
\hline Anti-Her2-f & AsnAib & 0.129 & 0.141 & 0.118 & 0.52 & 0.648 & 0.410 \\
\hline Anti-Her2-g & ValCit & 0.026 & 0.029 & 0.024 & 1.45 & 1.987 & 1.058 \\
\hline Anti-CD20-a & AsnAsn & 0.893 & 1.18 & 0.68 & 0.203 & 0.361 & 0.114 \\
\hline Anti-CD20-b & AsnAla & 1.24 & 1.46 & 1.05 & 0.180 & NA & NA \\
\hline Anti-CD20-c & GlnAsn & 0.936 & 1.03 & 0.85 & 0.149 & 0.161 & 0.138 \\
\hline Anti-CD20-d & GlnVal & 1.34 & 1.49 & 1.21 & 0.272 & 0.324 & 0.229 \\
\hline Anti-CD20-e & TyrTrp & 1.45 & 1.51 & 1.39 & 0.290 & 0.316 & 0.266 \\
\hline Anti-CD20-f & AsnAib & 1.18 & 1.30 & 1.07 & 0.334 & 0.368 & 0.303 \\
\hline Anti-CD20-g & ValCit & 2.46 & 2.77 & 2.18 & 0.084 & 0.087 & 0.081 \\
\hline
\end{tabular}

Table S8. Initial Enzymatic Velocities and Regression Analysis of Catabolic Enzymes against Linkers

\begin{tabular}{|c|c|c|c|c|c|c|c|c|}
\hline & \multicolumn{2}{|c|}{ Cathepsin B } & \multicolumn{2}{|c|}{ Tritosomes } & \multicolumn{2}{|c|}{ Legumain } & \multicolumn{2}{|c|}{ Ces1C } \\
\hline $\begin{array}{l}\text { Linker } \\
\text { Chemistry } \\
\text { (AA1-AA2) }\end{array}$ & $\begin{array}{c}\mathrm{V}_{0} \\
(\mathrm{nmol} / \mathrm{min})\end{array}$ & $\mathrm{R}$ & $\begin{array}{c}\mathrm{V}_{0} \\
(\mathrm{nmol} / \mathrm{min})\end{array}$ & $\mathrm{R}$ & $\begin{array}{c}\mathrm{V}_{0} \\
(\mathrm{nmol} / \mathrm{min})\end{array}$ & $R$ & $\begin{array}{c}\mathrm{V}_{0} \\
(\mathrm{nmol} / \mathrm{min})\end{array}$ & $\mathrm{R}$ \\
\hline ValCit & $5.82 \mathrm{E}-05$ & 0.999 & $6.62 \mathrm{E}-05$ & 0.999 & 8.39E-07 & 0.959 & $1.78 \mathrm{E}-05$ & 0.936 \\
\hline D-Val-D-Cit & 5.69E-06 & 0.962 & $8.82 \mathrm{E}-06$ & 0.949 & 1.19E-06 & 0.906 & $2.22 \mathrm{E}-05$ & 0.980 \\
\hline AlaAla & $2.76 \mathrm{E}-05$ & 0.977 & $2.64 \mathrm{E}-05$ & 0.949 & $2.01 \mathrm{E}-06$ & 0.849 & $3.83 \mathrm{E}-05$ & 0.974 \\
\hline GlyGly & $6.35 \mathrm{E}-06$ & 0.986 & $8.69 \mathrm{E}-06$ & 0.943 & $1.39 \mathrm{E}-06$ & 0.904 & 0.000193 & 0.999 \\
\hline GlyAsn & $8.66 \mathrm{E}-06$ & 0.989 & $1.26 \mathrm{E}-05$ & 0.947 & $1.06 \mathrm{E}-06$ & 0.790 & 0.000147 & 1.000 \\
\hline GlyAib & $1.06 \mathrm{E}-05$ & 0.966 & $1.3 \mathrm{E}-05$ & 0.893 & $1.81 \mathrm{E}-06$ & 0.836 & 0.000124 & 0.994 \\
\hline Gly1nal & $7.69 \mathrm{E}-06$ & 0.985 & $8.34 \mathrm{E}-06$ & 0.933 & $2.94 \mathrm{E}-06$ & 0.862 & 0.00019 & 0.998 \\
\hline Gly2nal & 7.97E-06 & 0.986 & 7.11E-06 & 0.916 & $2.94 \mathrm{E}-06$ & 0.826 & 0.000226 & 0.979 \\
\hline GlyPhe & $7.58 \mathrm{E}-06$ & 0.926 & $1.26 \mathrm{E}-05$ & 0.850 & $1.29 \mathrm{E}-05$ & 0.931 & 0.000121 & 1.000 \\
\hline GlyHis & $4.52 \mathrm{E}-06$ & 0.975 & $6.71 \mathrm{E}-06$ & 0.904 & $6.25 \mathrm{E}-07$ & 0.797 & $3.39 \mathrm{E}-05$ & 0.939 \\
\hline GlyVal & $9.22 \mathrm{E}-06$ & 0.906 & $1.35 \mathrm{E}-05$ & 0.871 & $2.36 \mathrm{E}-06$ & 0.797 & $9.26 \mathrm{E}-05$ & 0.980 \\
\hline GlyLys & $1.42 \mathrm{E}-05$ & 0.941 & $3.92 \mathrm{E}-05$ & 0.965 & $8.59 \mathrm{E}-07$ & 0.618 & $-4.7 \mathrm{E}-06$ & -0.300 \\
\hline GlyCit & $1.03 \mathrm{E}-05$ & 0.941 & $1.25 \mathrm{E}-05$ & 0.866 & $2.09 \mathrm{E}-06$ & 0.791 & $7.75 \mathrm{E}-05$ & 0.964 \\
\hline GlyTrp & $6.74 \mathrm{E}-06$ & 0.873 & 4.47E-06 & 0.515 & $2.5 \mathrm{E}-06$ & 0.619 & $2.04 \mathrm{E}-05$ & 0.928 \\
\hline SerAsn & $3.53 \mathrm{E}-06$ & 0.968 & $4.32 \mathrm{E}-05$ & 0.998 & $1.02 \mathrm{E}-05$ & 0.994 & $7.73 \mathrm{E}-05$ & 0.989 \\
\hline SerAib & $1.88 \mathrm{E}-06$ & 0.932 & $3.28 \mathrm{E}-06$ & 0.867 & $3.52 \mathrm{E}-07$ & 0.572 & $6.32 \mathrm{E}-05$ & 0.996 \\
\hline SerPhe & $1.86 \mathrm{E}-06$ & 0.886 & $2.75 \mathrm{E}-06$ & 0.637 & 9.99E-07 & 0.813 & $2.84 \mathrm{E}-05$ & 0.982 \\
\hline
\end{tabular}




\begin{tabular}{|c|c|c|c|c|c|c|c|c|}
\hline SerHis & $-9.6 \mathrm{E}-07$ & -0.292 & $-2.5 E-06$ & -0.558 & $1.15 \mathrm{E}-06$ & 0.917 & 4.53E-05 & 0.947 \\
\hline SerAla & $1.07 \mathrm{E}-05$ & 0.996 & $6.77 \mathrm{E}-06$ & 0.895 & 8.19E-07 & 0.906 & $7.16 \mathrm{E}-05$ & 0.963 \\
\hline SerVal & $6.08 \mathrm{E}-07$ & 0.418 & 1.19E-06 & 0.396 & 5.44E-07 & 0.873 & $3.32 \mathrm{E}-05$ & 0.986 \\
\hline SerLys & $-2.7 \mathrm{E}-06$ & -0.388 & $-6.7 \mathrm{E}-06$ & -0.650 & $6.16 \mathrm{E}-07$ & 0.525 & $2.59 \mathrm{E}-05$ & 0.972 \\
\hline SerCit & 7.94E-06 & 0.993 & $8.21 \mathrm{E}-06$ & 0.958 & $5.84 \mathrm{E}-07$ & 0.889 & $5.93 \mathrm{E}-05$ & 0.990 \\
\hline SerTrp & $6.76 \mathrm{E}-06$ & 0.994 & $5.12 \mathrm{E}-06$ & 0.951 & $7.33 \mathrm{E}-07$ & 0.941 & 0.000272 & 1.000 \\
\hline ProAsn & $4.36 \mathrm{E}-06$ & 0.990 & $9.28 \mathrm{E}-06$ & 0.944 & $1.21 \mathrm{E}-06$ & 0.945 & $6.76 \mathrm{E}-05$ & 0.998 \\
\hline ProAib & $2.52 \mathrm{E}-06$ & 0.826 & $6.54 \mathrm{E}-06$ & 0.880 & $3.36 \mathrm{E}-07$ & 0.685 & 3.77E-05 & 0.975 \\
\hline ProPhe & $2.25 \mathrm{E}-06$ & 0.957 & $2.88 \mathrm{E}-06$ & 0.734 & $1.07 \mathrm{E}-06$ & 0.899 & $5.24 \mathrm{E}-05$ & 0.964 \\
\hline ProHis & $1.92 \mathrm{E}-06$ & 0.912 & $2.74 \mathrm{E}-06$ & 0.763 & 8.07E-07 & 0.953 & $6.92 \mathrm{E}-05$ & 0.950 \\
\hline ProAla & $2.61 \mathrm{E}-06$ & 0.948 & $3.31 \mathrm{E}-06$ & 0.782 & $6.01 \mathrm{E}-07$ & 0.917 & 4.34E-05 & 0.986 \\
\hline ProVal & $2.72 \mathrm{E}-07$ & 0.315 & $1.95 \mathrm{E}-06$ & 0.736 & $4.9 \mathrm{E}-07$ & 0.844 & 3.09E-05 & 0.984 \\
\hline ProLys & $4.16 \mathrm{E}-07$ & 0.307 & $-1.5 \mathrm{E}-06$ & -0.448 & $9.62 \mathrm{E}-07$ & 0.921 & $4.74 \mathrm{E}-05$ & 0.981 \\
\hline ProCit & $1.14 \mathrm{E}-06$ & 0.805 & $2.55 \mathrm{E}-06$ & 0.728 & $8.08 \mathrm{E}-07$ & 0.925 & $3.38 \mathrm{E}-05$ & 0.980 \\
\hline ProTrp & $3.45 \mathrm{E}-06$ & 0.958 & $6.79 \mathrm{E}-06$ & 0.941 & $1.46 \mathrm{E}-06$ & 0.939 & 0.000398 & 1.000 \\
\hline HisAsn & 5.87E-07 & 0.589 & $3.32 \mathrm{E}-06$ & 0.861 & $1.36 \mathrm{E}-06$ & 0.980 & $5.39 \mathrm{E}-05$ & 0.994 \\
\hline HisAib & $3.45 \mathrm{E}-07$ & 0.395 & 5.03E-07 & 0.196 & $7.02 \mathrm{E}-07$ & 0.880 & $5.43 \mathrm{E}-05$ & 0.989 \\
\hline HisPhe & $1.97 \mathrm{E}-06$ & 0.877 & $1.02 \mathrm{E}-06$ & 0.272 & $1.7 \mathrm{E}-06$ & 0.891 & $4.04 \mathrm{E}-05$ & 0.973 \\
\hline HisAla & $7.55 \mathrm{E}-06$ & 0.990 & 5.69E-06 & 0.860 & $7.16 \mathrm{E}-07$ & 0.905 & $2.52 \mathrm{E}-05$ & 0.930 \\
\hline HisVal & $4.74 \mathrm{E}-06$ & 0.986 & $4.65 \mathrm{E}-06$ & 0.837 & $1.54 \mathrm{E}-06$ & 0.894 & $2.28 \mathrm{E}-05$ & 0.948 \\
\hline HisCit & $3.01 \mathrm{E}-06$ & 0.967 & 3.87E-07 & 0.157 & $7.62 \mathrm{E}-07$ & 0.928 & 1.39E-05 & 0.967 \\
\hline HisTrp & $2.76 \mathrm{E}-06$ & 0.937 & $1.21 \mathrm{E}-06$ & 0.407 & $1.39 \mathrm{E}-06$ & 0.858 & 0.000198 & 1.000 \\
\hline TyrAsn & $5.3 \mathrm{E}-06$ & 0.988 & $3 \mathrm{E}-05$ & 0.994 & $6.16 \mathrm{E}-06$ & 0.996 & $6.04 \mathrm{E}-05$ & 0.984 \\
\hline TyrAib & $3.82 \mathrm{E}-07$ & 0.445 & $2.01 \mathrm{E}-06$ & 0.613 & 7.64E-07 & 0.848 & $8.49 \mathrm{E}-05$ & 0.994 \\
\hline TyrPhe & 4.61E-06 & 0.989 & 8.16E-05 & 0.999 & 1.67E-06 & 0.856 & 4.66E-05 & 0.981 \\
\hline TyrPhe & $7.3 \mathrm{E}-06$ & 0.973 & $2.14 \mathrm{E}-05$ & 0.984 & $1.21 \mathrm{E}-06$ & 0.880 & $3.77 E-05$ & 0.969 \\
\hline TyrAla & $4.15 \mathrm{E}-05$ & 0.999 & 5.54E-05 & 0.996 & $6.89 \mathrm{E}-07$ & 0.870 & $3.53 \mathrm{E}-05$ & 0.985 \\
\hline TyrVal & $-1 \mathrm{E}-06$ & -0.557 & $6.34 \mathrm{E}-06$ & 0.853 & 9.01E-07 & 0.885 & 2.13E-05 & 0.970 \\
\hline TyrLys & $1.86 \mathrm{E}-06$ & 0.420 & $3.65 \mathrm{E}-06$ & 0.374 & 9.67E-07 & 0.614 & $7.85 \mathrm{E}-06$ & 0.965 \\
\hline TyrCit & $2.91 \mathrm{E}-05$ & 0.995 & $5.34 \mathrm{E}-05$ & 0.996 & $8.63 \mathrm{E}-07$ & 0.906 & $2.76 \mathrm{E}-05$ & 0.983 \\
\hline TyrTrp & 1.77E-05 & 0.997 & 0.000164 & 0.999 & $1.52 \mathrm{E}-06$ & 0.925 & 0.000476 & 1.000 \\
\hline GInAsn & 4.47E-06 & 0.970 & $2.13 \mathrm{E}-05$ & 0.997 & $5.45 \mathrm{E}-06$ & 0.992 & $5.73 \mathrm{E}-05$ & 0.995 \\
\hline GInAib & $1.46 \mathrm{E}-06$ & 0.881 & $2.31 \mathrm{E}-06$ & 0.707 & $8.15 E-07$ & 0.936 & $7 E-05$ & 0.997 \\
\hline GlnPhe & $2.39 \mathrm{E}-06$ & 0.919 & $4.68 \mathrm{E}-06$ & 0.883 & 9.27E-07 & 0.889 & $4.16 \mathrm{E}-05$ & 0.994 \\
\hline GlnHis & $1.72 \mathrm{E}-06$ & 0.909 & $2.38 \mathrm{E}-06$ & 0.728 & 9.47E-07 & 0.938 & 5.83E-05 & 0.956 \\
\hline GInAla & $8.25 \mathrm{E}-07$ & 0.392 & $-1.8 \mathrm{E}-07$ & -0.041 & $5.1 \mathrm{E}-07$ & 0.831 & 3.74E-05 & 0.964 \\
\hline GlnVal & $-4.9 \mathrm{E}-06$ & -0.911 & $-4.3 \mathrm{E}-06$ & -0.736 & 9.99E-07 & 0.896 & $1.41 \mathrm{E}-05$ & 0.969 \\
\hline GInLys & $-1 \mathrm{E}-05$ & -0.877 & $-1.8 \mathrm{E}-05$ & -0.938 & $1.84 \mathrm{E}-06$ & 0.829 & $4.75 \mathrm{E}-05$ & 0.966 \\
\hline GlnCit & $2.99 \mathrm{E}-06$ & 0.920 & 5.95E-06 & 0.787 & 7.69E-07 & 0.891 & $3.15 E-05$ & 0.982 \\
\hline GInTrp & $3.72 \mathrm{E}-06$ & 0.971 & 6.07E-06 & 0.949 & $1.11 \mathrm{E}-06$ & 0.972 & $9.25 \mathrm{E}-05$ & 0.935 \\
\hline IleAsn & $6.33 \mathrm{E}-06$ & 0.985 & 4.19E-05 & 0.996 & $1.3 \mathrm{E}-05$ & 0.986 & $8.27 \mathrm{E}-05$ & 0.998 \\
\hline IleAib & $2.19 \mathrm{E}-06$ & 0.881 & 5.65E-06 & 0.945 & $6.39 \mathrm{E}-07$ & 0.956 & 6.04E-05 & 0.994 \\
\hline llePhe & 1.62E-05 & 0.991 & $2.54 \mathrm{E}-05$ & 0.984 & $2.58 \mathrm{E}-06$ & 0.912 & 9.81E-05 & 0.972 \\
\hline IleHis & 5.06E-06 & 0.935 & 7.06E-06 & 0.962 & $9.58 \mathrm{E}-07$ & 0.928 & 5.89E-05 & 0.942 \\
\hline IleAla & $5.38 \mathrm{E}-05$ & 0.990 & 4.43E-05 & 0.998 & $5.55 \mathrm{E}-07$ & 0.939 & 4.97E-05 & 0.991 \\
\hline IleVal & $2.12 \mathrm{E}-06$ & 0.861 & $5.02 \mathrm{E}-06$ & 0.958 & 5.57E-07 & 0.897 & $4.52 \mathrm{E}-05$ & 0.989 \\
\hline
\end{tabular}




\begin{tabular}{|c|l|l|l|l|l|l|l|l|}
\hline IleLys & $1.31 \mathrm{E}-06$ & 0.159 & $-9 \mathrm{E}-06$ & -0.775 & $1.61 \mathrm{E}-06$ & 0.841 & $4.98 \mathrm{E}-05$ & 0.924 \\
\hline IleCit & $3.09 \mathrm{E}-05$ & 0.993 & $3.94 \mathrm{E}-05$ & 0.993 & $6.65 \mathrm{E}-07$ & 0.842 & $6.68 \mathrm{E}-05$ & 0.997 \\
\hline IleTrp & $1.28 \mathrm{E}-05$ & 0.993 & $3.58 \mathrm{E}-05$ & 0.998 & $1.38 \mathrm{E}-06$ & 0.901 & 0.000458 & 1.000 \\
\hline AsnAsn & $2.78 \mathrm{E}-07$ & 0.371 & 0.000325 & 1.000 & $9.34 \mathrm{E}-05$ & 0.997 & $8.45 \mathrm{E}-05$ & 1.000 \\
\hline AsnAib & $-4.6 \mathrm{E}-07$ & -0.524 & $1.43 \mathrm{E}-06$ & 0.559 & $6.3 \mathrm{E}-07$ & 0.936 & $7.56 \mathrm{E}-05$ & 0.997 \\
\hline AsnPhe & $1.96 \mathrm{E}-06$ & 0.941 & 0.000272 & 0.999 & $4.62 \mathrm{E}-05$ & 0.993 & $5.8 \mathrm{E}-05$ & 0.994 \\
\hline AsnHis & $3.27 \mathrm{E}-06$ & 0.977 & 0.00011 & 1.000 & $1.92 \mathrm{E}-05$ & 0.998 & $5.53 \mathrm{E}-05$ & 0.991 \\
\hline AsnAla & $1.27 \mathrm{E}-05$ & 0.981 & 0.000284 & 0.999 & $6.32 \mathrm{E}-05$ & 0.996 & $5.66 \mathrm{E}-05$ & 0.993 \\
\hline AsnVal & $2.22 \mathrm{E}-06$ & 0.948 & 0.000124 & 0.999 & $4.8 \mathrm{E}-05$ & 0.996 & $2.01 \mathrm{E}-05$ & 0.869 \\
\hline AsnLys & $8.83 \mathrm{E}-06$ & 0.957 & 0.000137 & 0.998 & $2.71 \mathrm{E}-05$ & 0.997 & $6.19 \mathrm{E}-05$ & 0.976 \\
\hline AsnCit & $8.18 \mathrm{E}-06$ & 0.966 & 0.000152 & 1.000 & $3.8 \mathrm{E}-05$ & 0.997 & $2.98 \mathrm{E}-05$ & 0.970 \\
\hline AsnTrp & $8.16 \mathrm{E}-06$ & 0.977 & 0.000227 & 1.000 & $2.73 \mathrm{E}-05$ & 0.995 & 0.00025 & 1.000 \\
\hline
\end{tabular}

\section{LCMS methods (analytical)}

Normal UPLC-MS Method: Analytical characterization was performed using a Water Acquity H-Class UPLC ${ }^{\circledR}$ with TUV detector, FLR detector, and QDa mass spectrometer. Typically, $1 \mu$ injections were separated using an Acquity UPLC BEH C18 $1.7 \mu \mathrm{m}$ column $(2.1 \times 50 \mathrm{~mm})$ at $80^{\circ} \mathrm{C}$. Eluent was monitored by UV (220 and $254 \mathrm{~nm})$, fluorescence (ex. $488 \mathrm{~nm}$, em. $520 \mathrm{~nm})$ and mass spectrometry (150-1250 Da, ES+/ES-). Solvents for the mobile phase were water with $0.1 \%$ formic acid (solvent $A$ ) and acetonitrile (ACN) with $0.1 \%$ formic acid (solvent B). Flow rate was $0.8 \mathrm{ml} / \mathrm{min}$. Gradient Used: Isocratic solvent B for $0.8 \mathrm{~min}(0-0.8 \mathrm{~min})$, then gradient from $10 \%-95 \%$ Solvent B over $4.2 \mathrm{~min}(0.8-4.5 \mathrm{~min})$, isocratic solvent $B$ for $0.3 \mathrm{~min}(4.5-4.8 \mathrm{~min})$, then gradient from $95 \%$ solvent $B$ over $0.1 \mathrm{~min}(4.8-4.9 \mathrm{~min})$, then isocratic solvent $B$ for $0.1 \mathrm{~min}(4.9-5.0 \mathrm{~min})$.

Polar UPLC-MS Method: Analytical characterization of hydrophilic, polar organic compounds was performed using an optimized UPLC gradient method. A Waters Acquity H-Class UPLC ${ }^{\circledR}$ with TUV detector and QDa Mass spectrometer was utilized. Typically, $1 \mu \mathrm{l}$ injections were separated using an Acquity UPLC BEH C18 $1.7 \mu \mathrm{m}$ column $(2.1 \times 50 \mathrm{~mm})$ at $80^{\circ} \mathrm{C}$. Eluent was monitored by UV ( 220 and $254 \mathrm{~nm}$ ), fluorescence (ex. $488 \mathrm{~nm}$, em. $520 \mathrm{~nm}$ ) and mass spectrometry (150-1250 Da, ES+/ES-). Solvents for the mobile phase were water with $0.1 \%$ formic acid (solvent $A$ ) and acetonitrile (ACN) with $0.1 \%$ formic acid (solvent B). Flow rate was $0.8 \mathrm{ml} / \mathrm{min}$. Gradient Used: Isocratic solvent B for $1.0 \mathrm{~min}$ (0$1.0 \mathrm{~min})$, then gradient from $3 \%$ to $50 \%$ Solvent B over $2.0 \mathrm{~min}(1.0-3.0 \mathrm{~min})$, then gradient from $50 \%$ to $95 \%$ solvent $B$ over $1.2 \mathrm{~min}$ (3.0-4.2 $\mathrm{min})$, then isocratic solvent $B$ for $0.1 \mathrm{~min}$ (4.2-4.3min), then gradient from $95 \%$ to $3 \%$ solvent $B$ for $0.1 \mathrm{~min}$ (4.3-4.4min), then isocratic solvent B for $0.6 \mathrm{~min}$ (4.4-5.0min).

Normal HPLC-MS Method: Analytical characterization of large (> $1250 \mathrm{Da}$ ) organic compounds was performed using a Waters Auto-purification system containing a 2545 binary gradient module, 2767 sample manager, 2998 UV/PDA detector, and SQD2 mass spectrometer. Typically, $1 \mu l$ injections were separated using an XBridge BEH C18 $5 \mu \mathrm{m}(4.6 \times 100 \mathrm{~mm})$ column at $80^{\circ} \mathrm{C}$. Eluent was monitored by UV (210-600 nm), and mass spectrometry (150-1800 Da, ES+/ES-). Solvents for the mobile phase were water with $0.05 \%$ formic acid (solvent $A$ ) and acetonitrile (ACN) with $0.05 \%$ formic acid (solvent B). Flow rate was $2.00 \mathrm{ml} / \mathrm{min}$. Gradient Used: Isocratic solvent $B$ for $1.0 \mathrm{~min}(0-1.0 \mathrm{~min})$, then gradient from $5 \%$ to $99 \%$ solvent B over $2.8 \mathrm{~min}$ (1.0-3.8min), then isocratic solvent B for $0.1 \mathrm{~min}$ (3.8-3.9min), then gradient from $99 \%$ to $5 \%$ solvent $B$ for $0.1 \mathrm{~min}$ (3.9-4.0 min), then isocratic solvent B for $1.0 \mathrm{~min}$ (4.0-5.0 min).

Normal Preparative HPLC Method: General purification of organic compounds was performed using a Waters Auto-purification system containing a 2545 binary gradient module, 2767 sample manager, 2998 
UV/PDA detector, and SQD2 mass spectrometer. Typically, 500 $\mu$ injections were separated using an XBridge BEH C18 $5 \mu \mathrm{m}$ OBD $(19 \times 100 \mathrm{~mm})$ prep column at room temperature. Eluent was monitored by UV (210-600nm), and mass spectrometry (150-2500 Da, ES+/ES-). Solvent for the mobile phase were water with $0.05 \%$ trifluoroacetic acid (solvent $A$ ) and acetonitrile $(A C N)$ with $0.05 \%$ trifluoroacetic acid (solvent B). Flow rate was $20.0 \mathrm{ml} / \mathrm{min}$. Gradient Used: Isocratic solvent B for $1.0 \mathrm{~min}(0-1.0 \mathrm{~min})$, then gradient from $15 \%$ to $95 \%$ solvent $B$ over $7.0 \mathrm{~min}(1.0-8.0 \mathrm{~min})$, then isocratic solvent B for $0.5 \mathrm{~min}(8.0$ $8.5 \mathrm{~min})$, then gradient from $95 \%$ to $15 \%$ solvent $B$ for $0.5 \mathrm{~min}(8.5-9.0 \mathrm{~min})$, then isocratic solvent $B$ for $1.0 \mathrm{~min}$ (9.0-10.0min) (Table IV).

Optimized Peptide Preparative HPLC Method: Purification of Oregon Green coupled linkers was optimized to separate closely eluting materials to product UV peaks that appeared under analytical traces. A Waters Auto-purification system containing a 2545 binary gradient module, 2767 sample manager, 2998 UV/PDA detector, and SQD2 mass spectrometer was employed. Typically, 500 1 injections were separated using an XBridge BEH C18 $5 \mu \mathrm{m}$ OBD $(19 \times 100 \mathrm{~mm})$ prep column at room temperature. Eluent was monitored by UV (210-600nm), and mass spectrometry (150-2500 Da, ES+/ES). Solvents for the mobile phase were water with $0.05 \%$ trifluoroacetic acid (solvent $A$ ) and acetonitrile (ACN) with $0.05 \%$ trifluoroacetic acid (solvent B). Flow rate was $20.0 \mathrm{ml} / \mathrm{min}$. Gradient Used: Isocratic solvent $B$ for $1.0 \mathrm{~min}(0-1.0 \mathrm{~min})$, then gradient from $5 \%$ to $20 \%$ solvent $B$ over $0.5 \mathrm{~min}$ (1.0-1.5min), then gradient from $20 \%$ to $50 \%$ solvent B over $7.5 \mathrm{~min}$ (1.5-8.0min), then gradient from $50 \%$ to $95 \%$ solvent $B$ for $0.5 \mathrm{~min}(8.0-8.5 \mathrm{~min})$, then gradient from $95 \%$ to $5 \%$ solvent $B$ for $0.5 \mathrm{~min}$ (8.5-9.0min), then isocratic solvent B for $1.0 \mathrm{~min}(9.0-10.0 \mathrm{~min})$.

LCMS method for ADC analysis: Analysis of ADCs was performed using a Water Autopurication system with a 2545 binary gradient module, 2767 sample manager, 2998 UV/PDA and a SQD2-mass spectrometer. Separation was performed using an Agilent Technologies poroshell 300 SB-C8 column $\left(2.1 \times 75 \mathrm{~mm}, 5 \mathrm{um}\right.$ ) at $80^{\circ} \mathrm{C}$ with a gradient of water $+0.05 \%$ formic acid (Buffer $A$ ) and acetonitrile $+0.05 \%$ formic acid (Buffer $B$ ). The gradient (flow rate $=1.00 \mathrm{~mL} / \mathrm{min}$ and $0.5 \mathrm{~mL} / \mathrm{min}$ ) was set up as follows:

\begin{tabular}{|l|l|l|l|}
\hline Time & Flow rate $(\mathrm{mL})$ & \%A & \% \\
\hline Initial & 1.00 & $95.0 \%$ & $5.0 \%$ \\
\hline 1.90 & 1.00 & $95.0 \%$ & $5.0 \%$ \\
\hline 2.00 & 1.00 & $5.0 \%$ & $95.0 \%$ \\
\hline 2.50 & 0.5 & $5.0 \%$ & $95.0 \%$ \\
\hline 4.00 & 0.5 & $5.0 \%$ & $95.0 \%$ \\
\hline 4.20 & 1.00 & $95.0 \%$ & $5.0 \%$ \\
\hline 5.00 & 1.00 & $95.0 \%$ & $5.0 \%$ \\
\hline
\end{tabular}

Samples ( $20 \mathrm{uL}$ of $\sim 1 \mathrm{mg} / \mathrm{mL}$ stock) were treated with $5 \mathrm{uL}$ of $0.5 \mathrm{M}$ TCEP immediately prior to analysis. The eluent was monitored by UV $(210 \mathrm{~nm}-600 \mathrm{~nm})$ and mass spec (ES+ $700-1800 \mathrm{Da}$. The antibody typically eluted at $3.9 \mathrm{~min}$. The raw charge envelope was deconvoluted using MaxEnt software. 